\title{
Nondestructive Detection of White Root Rot Disease in Avocado Rootstocks by Leaf Chlorophyll Fluorescence
}

\author{
E. Martínez-Ferri, A. Zumaquero, M. T. Ariza, A. Barceló, and C. Pliego, IFAPA, Centro de Churriana, Cortijo de la Cruz s/n, 29140 \\ Churriana, Málaga, Spain
}

\begin{abstract}
Martínez-Ferri, E., Zumaquero, A., Ariza, M. T., Barceló, A., and Pliego, C. 2016. Nondestructive detection of white root rot disease in avocado rootstocks by leaf chlorophyll fluorescence. Plant Dis. 100:49-58.

White root rot (WRR) disease caused by Rosellinia necatrix is one of the most important threats affecting avocado orchards in temperate regions. In this study, we monitored the progression of WRR disease at the leaf and root levels by the combination of nondestructive chlorophyll fluorescence measurements and confocal laser-scanning microscopy on avocado genotypes susceptible to $R$. necatrix. Leaf photochemistry was affected at early stages of disease development prior to the appearance of aboveground symptoms, made evident as significant decreases in the trapping efficiency of photosystem-II $\left(F_{\mathrm{v}}{ }^{\prime} / F_{\mathrm{m}}{ }^{\prime}\right)$ and in the steadystate of chlorophyll fluorescence yield $\left(F_{\mathrm{s}}\right)$ normalized to the minimal

fluorescence yield $\left(F_{0}\right)\left(F_{\mathrm{s}} / F_{0}\right)$. Decreases in $F_{\mathrm{v}}{ }^{\prime} / F_{\mathrm{m}}{ }^{\prime}$ and $F_{\mathrm{s}} / F_{0}$ were associated with different degrees of fungal penetration, primarily in the lateral roots but not in areas next to the main root collar. Aboveground symptoms were observed only when the fungus reached the root collar Leaf physiology was also tracked in a tolerant genotype where no changes were observed during disease progression despite the presence of the fungus in the root system. These results highlight the usefulness of this technique for the early detection of fungal infection and the rapid removal of highly susceptible genotypes in rootstock avocado-breeding programs
\end{abstract}

Diseases associated with fungal root invasion are relevant on woody crops (Pliego et al. 2012). Avocado (Persea americana Mill.) is a member of the Lauraceae family and an important commercial fruit crop in over 50 countries. One of the most important diseases affecting avocado orchards in temperate regions is white root rot (WRR), caused by the fungus Rosellinia necatrix Berl. ex Prill. (Freeman and Sztejnberg 1992; Pliego et al. 2012). Affected trees show root rotting followed by leaf wilting, eventually resulting in death of the trees after the appearance of the first foliar symptoms.

WRR disease diagnosis is a major problem for nurseries and commercial orchards. It requires high-throughput genetic techniques, such as those based on real-time Scorpion polymerase chain reaction (PCR; Ruano-Rosa et al. 2007; Schena and Ippolito 2003; Schena et al. 2002) that are not fully reliable in practice and require highly skilled personnel.

Even with an effective diagnosis, control of avocado WRR is a complex and difficult task as a consequence of the pathogen's features, including resistance to drought, survival capacity in acidic soils, colonization of numerous hosts, deep penetration into the soil, and immunity to various common fungicides. Current control approaches involve the use of physical (López-Herrera et al. 1999; Raj and Sharma 2009) and chemical (López-Herrera and Zea-Bonilla 2007; Sugimoto 2002) methods as well as biocontrol strategies (ten Hoopen and Krauss 2006), although none of these methods has proven to be fully effective; hence, the development of more reliable tools to control this disease is needed (Pliego et al. 2012). As in other woody crops, the use of disease-tolerant or -resistant rootstocks would constitute a method for widespread control pathogen (Broadbent and Gollnow 1993; Dadmal et al. 2002; Trapero et al. 2008; Verdejo-Lucas et al. 2003). Until recently, no commercial rootstocks showing tolerance to $R$. necatrix have been available (Pliego et al. 2012), although several promising selections are being evaluated (Barceló-Muñoz et al. 2007) (A. Barceló-Muñoz, IFAPA, Centro de

Corresponding author: C. Pliego; E-mail: mclara.pliego@juntadeandalucia.es Accepted for publication 25 June 2015.

http://dx.doi.org/10.1094/PDIS-01-15-0062-RE

(C) 2016 The American Phytopathological Society
Churriana, Málaga, Spain, personal communication). However, success of breeding programs is greatly limited by the time-consuming and difficult process of working with woody plants. In this sense, the development of rapid and sensitive methods for $R$. necatrix detection would permit substantial advances in breeding for tolerant rootstocks.

Understanding the physiological changes in the avocado- $R$. necatrix pathosystem during the first stages of disease development is necessary for the development of a rapid and comprehensive detection method for WRR disease (Bauriegel and Herppich 2014). Some specialized root pathogens, such as Phytophthora spp., cause root rot and leaf physiology impairments prior to wilting (Fleischmann et al. 2002, 2004). These studies also observed that the fungal invasion of the plant root system impairs water uptake and flow, inducing water stress; which, in part, is counteracted through stomatal closure at the expense of limiting photosynthesis. Infection by some soilborne pathogens triggered a systemic effect on photosynthesis associated with the release of toxic mobile metabolites or hormonal imbalances (Brummer et al. 2002).

$R$. necatrix simultaneously invades avocado at several points within the roots, and invasion is followed by the proliferation of hyphal strands in one or several directions, invading both epidermal and cortical cells and, finally, collapsing the vascular system of the plant (Pliego et al. 2009). R. necatrix produces several phytotoxic compounds in vitro, which have been associated with pathogenicity and virulence (Edwards et al. 2001; Kimura et al. 1989; Kshirsagar et al. 2001). However, it is not clear whether WRR symptomatology results from these phytotoxic components or the mycelial invasion of vascular root tissues (Pliego et al. 2012). Thus, it is reasonable to expect that $R$. necatrix would induce a response at the leaf level as the disease progresses. As for other pathogens, nondestructive techniques based on measurements of chlorophyll fluorescence and chlorophyll content (SPAD) could be useful for the early detection of this disease (Bauriegel and Herppich 2014; Berger et al. 2007a; Calderón et al. 2014).

The main aims of the present study were to (i) assess whether the response of susceptible avocado plants at early stages of $R$. necatrix root infection were associated with changes at the physiological leaf level prior to the appearance of any visible symptoms and, if true, (ii) determine whether this response occurs in genotypes with different degrees of tolerance. 
The physiological changes in avocado leaves during the first stages of $R$. necatrix infection were evaluated using a combination of nondestructive chlorophyll fluorescence measurements and confocal laser microscopy to visualize the fungal invasion of the plant root system.

\section{Material and Methods}

Fungal isolates and culture conditions. The fungal strains used in this study were the virulent CH53 strain, isolated in an avocado orchard at Almuñecar (Granada, Spain) in 1991 (López-Herrera et al. 1999), and the virulent derivative strain CH53-gfp, expressing the green fluorescent protein (GFP), obtained through protoplast transformation (Pliego et al. 2009). Fungal strains were grown at $25^{\circ} \mathrm{C}$ on potato dextrose agar (PDA; Difco Laboratories). The routine growth of the $g f p$-derivate strain was performed on media containing hygromicine $(\mathrm{HygB})$ at $50 \mu \mathrm{g} / \mathrm{ml}$.

Plant material and pathogenicity test in avocado plants. Pathogenicity tests were performed in avocado genotypes differing in their disease reaction to $R$. necatrix. Three complementary experiments were carried out.

Experiment 1. Plantlets of three different 'Topa-Topa' avocado genotypes (TT-16, TT-21, and TT-31) were tested for assessing the early response to $R$. necatrix infection. These genotypes are commonly used as susceptible to $R$. necatrix in the ongoing breeding program for selection of tolerant rootstocks. The three genotypes were established in vitro and micropropagated according to the protocol of PliegoAlfaro et al. (2013). In vitro rooted shoots from each plantlet were acclimated in seedbeds filled with a mixture of peat, coconut fiber, and perlite at a ratio of 10:10:1 and $10 \mathrm{ml}$ of Osmocote under 100\% humidity. After 45 days, the plants were potted in 1.5 -liter plastic pots containing the same substrate and placed in a lathhouse for 6 months.

Prior to inoculation, plantlets were potted in 3-liter plastic pots (14 cm in diameter by $20 \mathrm{~cm}$ high) and transferred to controlled greenhouse conditions (approximately $20^{\circ} \mathrm{C}$ and $60 \%$ relative humidity $[\mathrm{RH}])$. The plants were placed on benches and distributed into six trays of eight plants per genotype and allowed to establish. Five clonal plants per genotype were used as noninoculated control plants.

From a total of eight inoculated plants for each genotype, five plants were inoculated with $R$. necatrix wild-type strain and the three remaining plants were inoculated with the $R$. necatrix-gfp derivative strain.

For genotype characterization, plant size measurements (plant height, stem diameter, and number of leaves) were obtained for control plants prior to inoculation (25 October 2013) and 9 weeks postinoculation (when most of the inoculated plants displayed aerial symptoms; 9 January 2014). In these plants, the biomass partitioning variables, including root/shoot ratio $(\mathrm{R} / \mathrm{S}$; root dry weight per shoot dry weight), root weight ratio (RWR; root dry weight per plant dry weight), and leaf weight ratio (LWR; leaf dry weight per plant dry weight) were calculated by weighing the stems, leaves, and roots of each plant separately before and after oven drying at $65^{\circ} \mathrm{C}$ for $48 \mathrm{~h}$. The shoot moisture content was estimated from the difference between the fresh and dry weights. The leaf fresh and dry weights were used to calculate the leaf water content (LWC) on a fresh weight basis ([fresh weight - dry weight]/fresh weight).

During this experiment, the temperature (T) and RH in the greenhouse were recorded every $30 \mathrm{~min}$ using an U23-001 HOBBO* Pro v2 logger (Onset Computer Corporation). The diurnal air $\mathrm{T}$ ranged from $20.9 \pm 0.6^{\circ} \mathrm{C}$ to $10.0 \pm 0.7^{\circ} \mathrm{C}$, and the mean $\mathrm{RH}$ was $61.2 \pm$ $1.8 \%$ during the course of the experiment. The plantlets were hand irrigated twice a week to ensure soil wetness.

Experiment 2. To validate results of experiment 1, plants of 'Dusa' avocado genotype, previously described as susceptible to $R$. necatrix, were tested for assessing the early response to $R$. necatrix infection. Vegetatively propagated plants (9 months old) of the commercial 'Dusa' rootstock (Brokaw nursery, Spain) were grown under controlled greenhouse conditions (approximately $20^{\circ} \mathrm{C}$ and approximately $60 \% \mathrm{RH}$ ) in 3-liter plastic pots ( $14 \mathrm{~cm}$ in diameter by $20 \mathrm{~cm}$ high) containing the same substrate mix described above. In total, 26 plants were used, but plants were distributed in two sets of 13 plants to conduct two trials (March to April 2014 and May to June 2014). In each trial, four plants were used as noninoculated (control plants) and the remaining plants were inoculated with $R$. necatrix. For genotype characterization, plant size measurements (plant height, stem diameter, and number of leaves) were recorded in all plants prior to first inoculation (20 March). Biomass partitioning variables were also estimated in control plants at the end of the experiment, as described above. Environmental conditions during the experimentation ( $\mathrm{T}$ and $\mathrm{RH})$ were also recorded with a U23-001 HOBBO* Pro v2 logger (Onset Computer Corporation). Diurnal air $\mathrm{T}$ ranged from $19.8 \pm 0.3$ to $11.7 \pm 0.3^{\circ} \mathrm{C}$ in trial 1 and from $23.3 \pm 0.3$ to $16.7 \pm 0.4^{\circ} \mathrm{C}$ in trial 2 . Mean RH was $66.7 \pm 1.5$ and $65.0 \pm$ $1.3 \%$ in the two trials, respectively. Plantlets were hand irrigated evenly to ensure soil wetness.

Experiment 3. To test the photochemical response to $R$. necatrix infection on a genotype described as tolerant to this pathogen in the ongoing avocado rootstock breeding program, plants of BG83 selection were used. Explants were established in vitro and micropropagated according to the protocol of Pliego-Alfaro et al. (2013). Once plants were acclimated in seedbeds, they were transferred to a lathhouse. When plants were one and a half years old, they were potted in 9-liter plastic pots ( $24 \mathrm{~cm}$ in diameter by $20 \mathrm{~cm}$ high) using the same substrate described above and transferred to controlled greenhouse conditions for inoculation. In total, 26 plants were tested in two trials conducted in March to May 2014 and May to July 2014. On each trial, four plants were used as noninoculated (control plants) and nine plants were inoculated with $R$. necatrix. Plant size measurements were recorded in all plants prior to the first inoculation (20 March) and biomass partitioning variables were also estimated in control plants at the end of the experiment.

Environmental conditions during the experimentation ( $\mathrm{T}$ and $\mathrm{RH}$ ) were also recorded with a U23-001 HOBBO* Pro v2 logger (Onset Computer Corporation). Diurnal air T ranged from $20.9 \pm 0.3$ to $12.6 \pm 0.3^{\circ} \mathrm{C}$ in trial 1 and from $24.1 \pm 0.2$ to $17.8 \pm 0.3^{\circ} \mathrm{C}$ in trial 2. Mean RH was $65.5 \pm 1.0 \%$ in the two trials. Plants were hand irrigated evenly to ensure soil wetness.

Pathogenicity assays were performed according to Sztejnberg and Madar (1980). The plants were inoculated with $3.75 \mathrm{~g}$ of colonized wheat seed per liter of substrate and monitored during disease progression. To assure the spread of the inoculum, it was placed on eight points scattered around the stem (approximately $3.5 \mathrm{~cm}$ apart) and introduced at two depths (at approximately 5 and $15 \mathrm{~cm}$, respectively). The aerial symptoms were observed and assessed daily according to a scale of $1=$ healthy plant, $2=$ mild wilting, $3=$ wilting, $4=$ desiccated, and $5=$ death.

Chlorophyll $\boldsymbol{a}$ fluorescence measurements. In vivo chlorophyll $a$ fluorescence signals were measured in all plants at midmorning (1000 to $1100 \mathrm{~h}$ ) daily or every 2 days in experiment 1 or twice a week in experiments 2 and 3. Measurements were taken using a portable fluorimeter PAM-2100 (Heinz Walz) equipped with leaf-clip holders to monitor incident light (photon flux density) and leaf T. On each plant, one (experiments 1 and 2) or two (in experiment 3) fully expanded mature leaves were labeled at the onset of the experiments for repeated measurements throughout the experimental courses on the same leaves. The so-called saturation pulse method was used for the determination of all fluorescence parameters (Schreiber et al. 1994). The dark-adapted parameters were determined at predawn (0600 to $0700 \mathrm{~h}$ ) prior to inoculation with $R$. necatrix. To assess the initial minimal fluorescence $\left(F_{0}\right)$ in the dark or the steady-state fluorescence $\left(F_{\mathrm{s}}\right)$ in the light (at approximately $450 \mu \mathrm{mol}$ quanta $\mathrm{m}^{-2} \mathrm{~s}^{-1}$ ), the leaf samples were exposed to a weakly modulated measuring beam. Subsequently, a saturating flash of light $\left(12,000 \mu \mathrm{mol}\right.$ quanta $\left.\mathrm{m}^{-2} \mathrm{~s}^{-1}\right)$ was administered for $0.8 \mathrm{~s}$ to assess the maximal fluorescence levels, either in the dark, when photosystem II (PSII) centers are closed $\left(F_{\mathrm{m}}\right)$, or under light conditions $\left(F_{\mathrm{m}}{ }^{\prime}\right)$. The leaves were immediately darkened after every saturation pulse and subsequently exposed to far-red light for $5.5 \mathrm{~s}$ to determine the minimal fluorescence yield of the preilluminated sample $\left(F_{0}{ }^{\prime}\right)$. Measurements of $F_{\mathrm{m}}$ and $F_{0}$ were obtained at predawn to calculate the maximal photochemical efficiency of PSII $\left(F_{\mathrm{v}} / F_{\mathrm{m}}=\left[F_{\mathrm{m}}-F_{0}\right] / F_{\mathrm{m}}\right)$ and the extent of Stern-Volmer nonphotochemical fluorescence quenching (NPQ $\left.=\left[F_{\mathrm{m}}-F_{\mathrm{m}}{ }^{\prime}\right] /\left[F_{\mathrm{m}}{ }^{\prime}\right]\right)$ (Bilger and Björkman 1990). The relative quantum yield of PSII 
photochemistry $\left(\Phi_{\mathrm{PSII}}=\left[F_{\mathrm{m}}{ }^{\prime}-F_{\mathrm{s}}\right] / F_{\mathrm{m}}{ }^{\prime}\right)$, photochemical quenching ( $\left.\mathrm{qP}=\left[F_{\mathrm{m}}{ }^{\prime}-F_{\mathrm{s}}\right] /\left[F_{\mathrm{m}}{ }^{\prime}-F_{0}{ }^{\prime}\right]\right)$, and maximum photochemical efficiency of the open reaction centers of PSII $\left(F_{\mathrm{v}}{ }^{\prime} / F_{\mathrm{m}}{ }^{\prime}=\left[F_{\mathrm{m}}{ }^{\prime}-F_{0}{ }^{\prime}\right] / F_{\mathrm{m}}{ }^{\prime}\right)$ were calculated according to Genty et al. (1989).

The steady-state fluorescence yield $\left(F_{\mathrm{s}}\right)$ was normalized to darkadapted fluorescence yields $\left(F_{0}\right) . F_{\mathrm{s}}$ has been described as a function of the competition between photochemical and nonphotochemical de-excitation of the energy absorbed by light-harvesting complexes (Schreiber et al. 1998).

SPAD index. A hand-held chlorophyll meter (SPAD 502; Minolta) was used to measure leaf greenness according to a unitless SPAD index ranging from 0 to 100 . This index has previously been used as an estimation of the chlorophyll content in the leaves (Jifon et al. 2005; Markwell et al. 1995; Uddling et al. 2007) associated with the degree of chlorosis (Tavakkoli et al. 2010). The measurements are based on the ratio of chlorophyll absorbance in two wavebands (red $=650 \mathrm{~nm}$, peak of chlorophyll absorbance, and infrared $=$ approximately $940 \mathrm{~nm}$, nonchlorophyll absorbance). The average SPAD values were calculated from three readings per leaf taken on the same leaves as for chlorophyll fluorescence measurements.

Confocal microscopy. Microscopy was assessed in experiment 1 . The roots of three avocado plants of each genotype were infected with $R$. necatrix CH53-gfp as described above. The infected roots of each plant were carefully removed after a sustained decrease in the parameter $F_{\mathrm{v}}{ }^{\prime} / F_{\mathrm{m}}{ }^{\prime}$ or $F_{\mathrm{s}} / F_{0}$ was observed, although the plant appeared to be healthy (Fig. 1, stage 1), and once the disease was well established (Fig. 1, stages 2 and 3). The roots were gently swirled in sterile water to wash away the sand particles. To assess mycelium colonization along the surface of avocado roots, the root segments were placed directly into petri dishes and observed under a fluorescence microscope (Leica Microsystems) equipped with filter blocks with spectral properties matching those of enhanced GFP (488 nm excitation and emission from 500 to $560 \mathrm{~nm}$ ).

To examine the internal proliferation and tissue invasion of $R$. necatrix, the avocado root fragments were fixed in agarose according to Alvarez et al. (2006). The samples were fixed overnight in a $2.5 \%$ paraformaldehyde solution with an increasing sucrose gradient of 10 , 20 , and $30 \%$ for 20,20 , and $30 \mathrm{~min}$, respectively. After treatment, samples were embedded in $7 \%$ low-melting-point agarose gel and stored at $4{ }^{\circ} \mathrm{C}$. The samples were subsequently sliced using a Leica 2000 microtome with freezing. Ultimately, 30- to $90-\mu \mathrm{m}$ sections were obtained and observed using confocal laser-scanning microscopy (CLSM; model TCS NT; Leica).

Statistical analyses. The data were analyzed using STATISTICA 7.0 analytical software (Statsoft Inc.). Differences in plant size and biomass partitioning variables among genotypes were evaluated using one-way analysis of variance (ANOVA). In experiment 1 , to evaluate the differences among treatments and genotypes in chlorophyll fluorescence variables and SPAD index throughout the experimental course, two-way repeated measures ANOVA (r-ANOVA) was performed. In this analysis, "genotype" and "treatment" were the between-subjects factors and "date" was the within-subjects factor. Genotypes that were not significantly different were joined to evaluate the treatment effect using one-way r-ANOVA. In experiments 2 and 3, treatment effects were also evaluated by one-way r-ANOVA. The pattern of disease response observed in the two trials of each experiment was similar and data from the two trials were analyzed either separately or jointly. To analyze single sampling dates, two-way ANOVA was used. Significant differences were considered at the 5\% probability level, unless otherwise stated. Prior to ANOVA, normality and homogeneity assumptions were tested using the Kolmogorov-Smirnov and Cochran's C tests, respectively. When significant differences were observed, Fisher's least significant difference test was used to compare the mean values.

\section{Results}

Plant characterization. Plant height, number of leaves, and stem diameter of all genotypes were measured at the beginning of each experiment. In experiment 1 , these measurements were also taken at the end. In experiment 1 , no significant differences between genotypes were observed in the stem diameter and number of leaves (Table 1). However, plant height was significantly lower in TT-21 than in TT-31 $(P=0.045$; Table 1$)$, although these differences were not observed at the end of the experiment, likely resulting from a faster growth rate in TT-21 plants, indicated as a significantly higher increase in the stem diameter and number of leaves (Table 1). Plant dry weight, biomass allocation pattern, and leaf water content were similar in all plants from the three Topa-Topa genotypes. The roots and leaves represented approximately 26 and 55\%, respectively, of the total plant dry weight. Plants ages in experiments 2 and 3 were different and, therefore, direct comparisons in size are not appropriate. However, plant size measurements indicated a different architecture in the tolerant genotype (i.e., more leaves per stem diameter; Table 1). Biomass allocation to roots was comparatively higher in BG83 than in the susceptible genotypes, which resulted in higher root/shoot ratios (Table 1).

Postinfestation development of aerial and physiological symptoms. The initial visible aboveground WRR symptoms (Fig. 1) were observed around 3 weeks after inoculation in TT-16, TT-21 (experiment 1), and Dusa (experiment 2) genotypes (Fig. 2A and C). At this time, only 20 to $30 \%$ of the plants were at stage 2 (mild wilting; Fig. 1, stage 2) although, in 1 week, the symptoms progressed to advanced stages of desiccation (Fig. 1, stages 3 and 4). Five weeks after inoculation, more than $60 \%$ of the plants were completely desiccated or dead (Fig. 1, stages 4 and 5). In contrast, initial disease symptoms in TT-31 (experiment 1) and BG83 plants (experiment 3) were delayed to more than 4 weeks after inoculation, and advanced stages of the disease appeared progressively within 8 weeks. Approximately more than $50 \%$ of plants were dead after 11 weeks postinoculation (Fig. 2B and D). At this time, plants that were alive in TT-31 displayed wilting symptoms (stage 3 ) and finally died in two more weeks

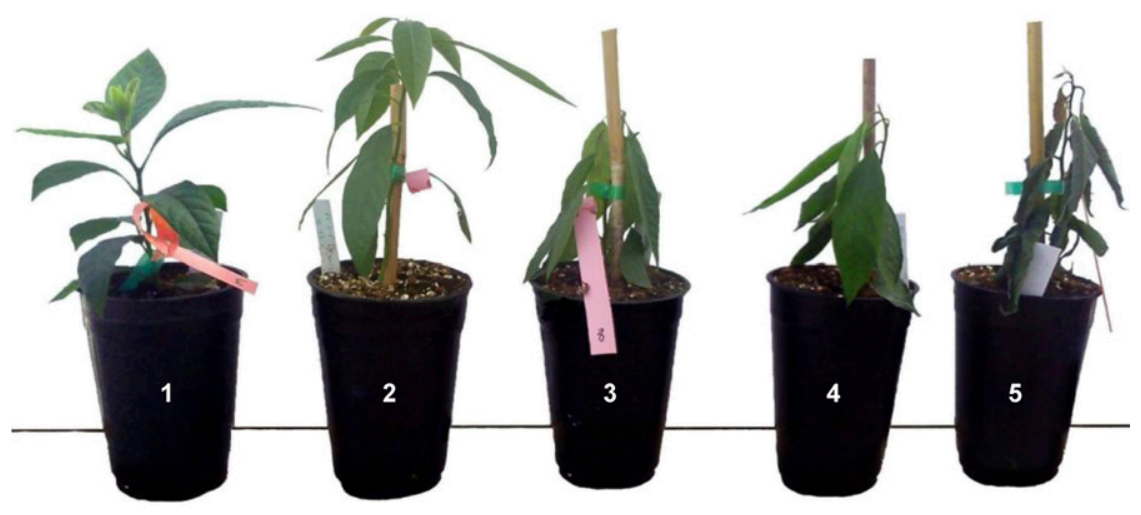

Fig. 1. Stages of aerial symptoms on avocado caused by white root rot: $1=$ healthy plant, $2=$ mild wilting, $3=$ wilting, $4=$ desiccated, and $5=$ death. 
whereas, in BG83, plants were completely healthy and remained alive, consistently with a tolerant disease reaction.

The dark-adapted $\mathrm{F}_{\mathrm{v}} / \mathrm{F}_{\mathrm{m}}$ values for all plants examined ranged from 0.797 to 0.808 (data not shown), indicating that plants were completely healthy at the beginning of the experiment. In susceptible genotypes, significant physiological changes were observed prior to the appearance of any aerial symptoms (Figs. 3 and 4). These changes occurred earlier in inoculated TT-16, TT-21, and Dusa plants. In these genotypes, a progressive decrease in the maximum photochemical efficiency of PSII open reaction centers $\left(F_{\mathrm{v}}{ }^{\prime} / F_{\mathrm{m}}{ }^{\prime}\right)$ and the steady-state yield of chlorophyll fluorescence $\left(\mathrm{F}_{\mathrm{s}}\right)$ normalized to $\mathrm{F}_{0}\left(\mathrm{~F}_{\mathrm{s}} / \mathrm{F}_{0}\right)$ was observed. In TT-16 and TT-21, differences on each parameter between both treatments were statistically significant at 10 to 15 days postinoculation, respectively (Fig. $3 \mathrm{~A}$ and $\mathrm{C}$; $\left.F_{\mathrm{v}}{ }^{\prime} / F_{\mathrm{m}}{ }^{\prime}, P=0.009 ; \mathrm{F}_{\mathrm{S}} / \mathrm{F}_{0}, P=0.033\right)$; whereas, in Dusa, statistical differences were noticeable after 21 days postinoculation (Fig. 4A and $\left.\mathrm{C} ; F_{\mathrm{v}}{ }^{\prime} / F_{\mathrm{m}}{ }^{\prime}, P=0.002 ; \mathrm{F}_{\mathrm{s}} / \mathrm{F}_{0}, P=0.023\right)$. No significant variation in $\mathrm{qP}$ or $\Phi_{\text {PSII }}$ was observed before the appearance of any visible aerial symptoms (data not shown). The decrease in $F_{\mathrm{v}}{ }^{\prime} / F_{\mathrm{m}}{ }^{\prime}$ and $\mathrm{F}_{\mathrm{s}} / \mathrm{F}_{0}$ was paralleled by a concomitant and significant increase in NPQ

Table 1. Plant size and biomass partitioning variables in control plants of the Topa-Topa, Dusa, and BG83 avocado genotypes ${ }^{\mathrm{Z}}$

\begin{tabular}{|c|c|c|c|c|c|}
\hline Variables & TT-16 & TT-21 & TT-31 & DUSA & BG83 \\
\hline \multicolumn{6}{|l|}{ Height $(\mathrm{cm})$} \\
\hline Beginning & $16.00 \pm 1.03 a b$ & $18.27 \pm 0.77 \mathrm{a}$ & $15.22 \pm 0.43 \mathrm{~b}$ & $62.97 \pm 1.31$ & $51.27 \pm 2.28$ \\
\hline End & $19.16 \pm 2.97$ & $22.88 \pm 1.53$ & $21.30 \pm 1.40$ & $\ldots$ & $\ldots$ \\
\hline Relative increase & $(0.20)$ & $(0.25)$ & $(0.40)$ & $\ldots$ & $\ldots$ \\
\hline \multicolumn{6}{|l|}{ Number of leaves } \\
\hline Beginning & $14.69 \pm 0.73$ & $12.70 \pm 0.83$ & $12.92 \pm 0.54$ & $14.00 \pm 1.15$ & $27.78 \pm 1.49$ \\
\hline End & $19.60 \pm 2.06$ & $21.50 \pm 1.26$ & $20.33 \pm 1.45$ & $\ldots$ & $\ldots$ \\
\hline Relative increase & $(0.33)$ & $(0.69)$ & $(0.57)$ & $\ldots$ & $\ldots$ \\
\hline \multicolumn{6}{|l|}{ Stem diameter $(\mathrm{mm})$} \\
\hline Beginning & $2.88 \pm 0.19$ & $2.81 \pm 0.22$ & $3.03 \pm 0.11$ & $9.31 \pm 0.16$ & $8.62 \pm 0.21$ \\
\hline End & $3.60 \pm 0.31$ & $4.72 \pm 0.29$ & $4.40 \pm 0.39$ & $\ldots$ & $\ldots$ \\
\hline Relative increase & $(0.25) \mathrm{b}$ & $(0.68) \mathrm{a}$ & $(0.45) \mathrm{b}$ & $\ldots$ & $\ldots$ \\
\hline Plant dry weight (g) & $4.03 \pm 0.83$ & $5.79 \pm 0.94$ & $6.48 \pm 1.32$ & $29.14 \pm 3.90$ & $57.79 \pm 1.95$ \\
\hline Leaf water content $(\%)$ & $68.01 \pm 0.50$ & $66.50 \pm 0.89$ & $68.10 \pm 1.05$ & $\ldots$ & $\ldots$ \\
\hline Leaf weight ratio ( $\mathrm{g} \mathrm{g}^{-1}$ plant) & $0.56 \pm 0.03$ & $0.51 \pm 0.04$ & $0.57 \pm 0.03$ & $0.37 \pm 0.02$ & $0.23 \pm 0.03$ \\
\hline Root weight ratio ( $\mathrm{g} \mathrm{g}^{-1}$ plant) & $0.26 \pm 0.02$ & $0.29 \pm 0.03$ & $0.26 \pm 0.02$ & $0.27 \pm 0.01$ & $0.40 \pm 0.02$ \\
\hline Root to shoot ratio $\left(\mathrm{g} \mathrm{g}^{-1}\right)$ & $0.35 \pm 0.04$ & $0.41 \pm 0.07$ & $0.35 \pm 0.04$ & $0.36 \pm 0.01$ & $0.66 \pm 0.07$ \\
\hline
\end{tabular}

A
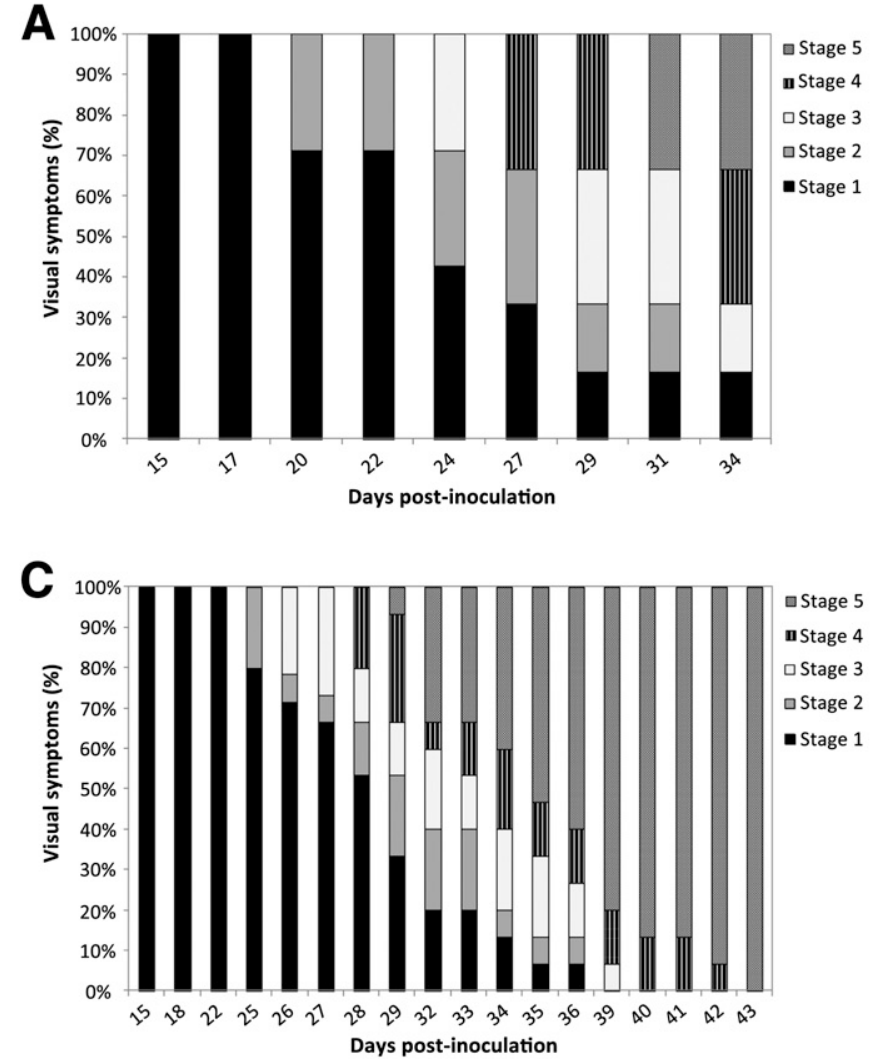

B
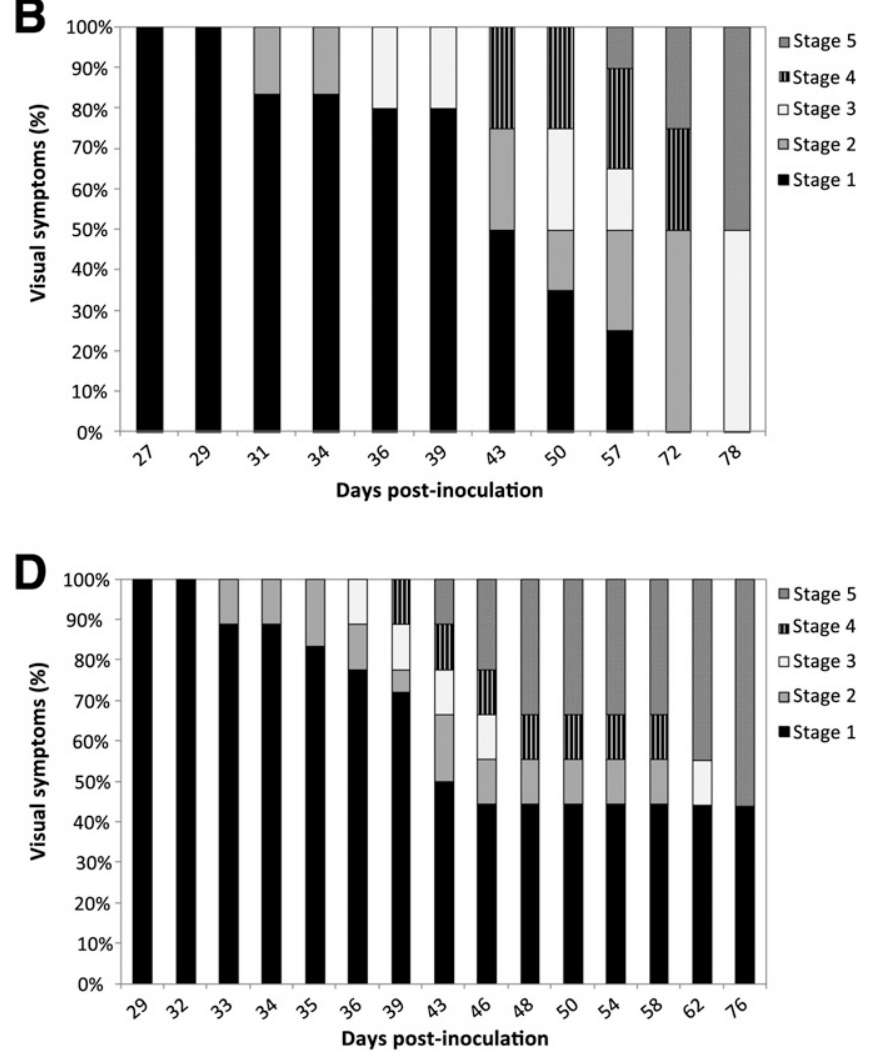

Fig. 2. Development of aerial symptoms (\%) throughout the experiments in the inoculated plants of A, TT-16 and TT-21; B; TT-31; C, 'Dusa'; and D, BG83 avocado genotypes. Stages: $1=$ healthy plant, $2=$ mild wilting, $3=$ wilting, $4=$ desiccated, and $5=$ death. 


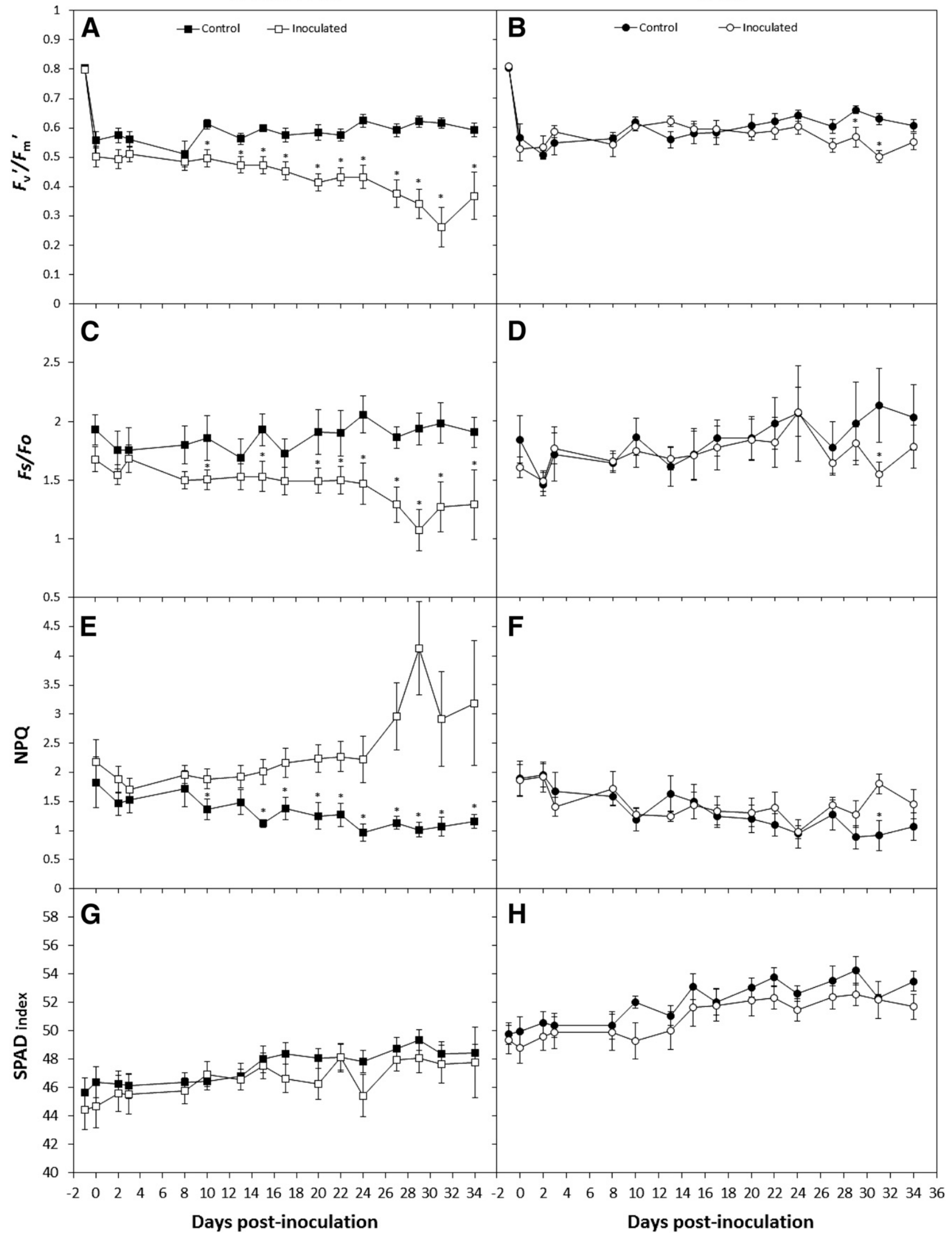

Fig. 3. Variation in the photochemical efficiency of the $\mathbf{A}$ and $\mathbf{B}$, open reaction centers of photosystem $\|\left(F_{\mathrm{v}}{ }^{\prime} / F_{\mathrm{m}}{ }^{\prime}\right) ; \mathbf{C}$ and $\mathbf{D}$, steady-state chlorophyll fluorescence yield $\left(F_{\mathrm{s}}\right)$ normalized to the minimal fluorescence yield $\left(F_{0}\right)\left(F_{s} / F_{0}\right) ; \mathbf{E}$ and $\mathbf{F}$, nonphotochemical quenching (NPQ); and $\mathbf{G}$ and $\mathbf{H}, \mathrm{SPAD}$ index throughout experiment 1 in the three 'Topa-Topa' avocado genotypes studied (TT-16, TT-21, and TT-31). TT-16 and TT-21 were joined in the same plot, because these plants showed similar disease reactions against the pathogen. Asterisks indicate significant differences between inoculated and control treatments $(P<0.05)$. Each data point represents the mean $( \pm$ standard error; $n=5$ to 16$)$. 


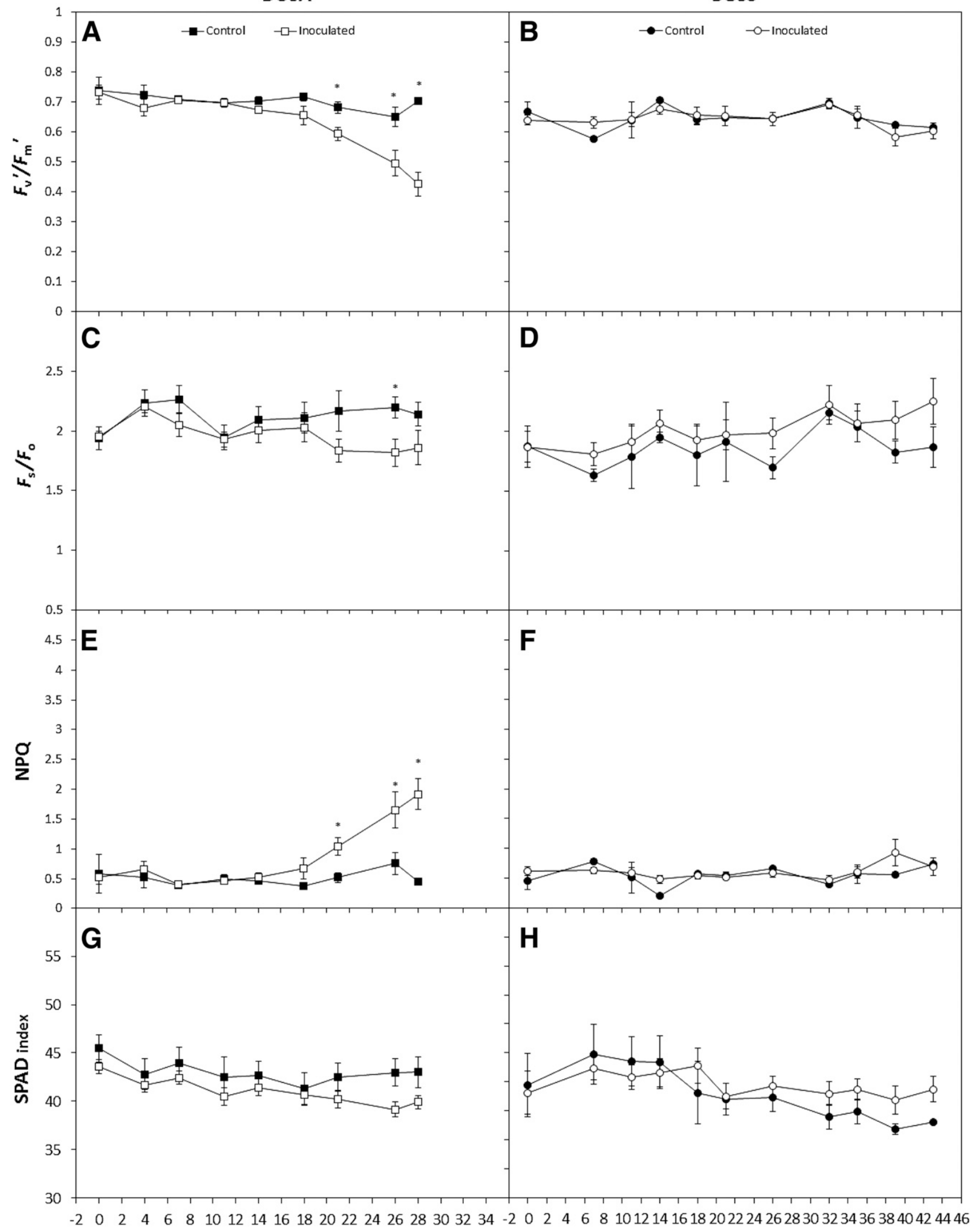

Days post-inoculation

Days post-inoculation

Fig. 4. Variation in the photochemical efficiency of the $\mathbf{A}$ and $\mathbf{B}$, open reaction centers of photosystem $\|\left(F_{\mathrm{v}}{ }^{\prime} / F_{\mathrm{m}}{ }^{\prime}\right) ; \mathbf{C}$ and $\mathbf{D}$, steady-state chlorophyll fluorescence yield $\left(F_{\mathrm{s}}\right)$ normalized to the minimal fluorescence yield $\left(F_{0}\right)\left(F_{s} / F_{0}\right) ; \mathbf{E}$ and $\mathbf{F}$, nonphotochemical quenching (NPQ); and $\mathbf{G}$ and $\mathbf{H}$, SPAD index in 'Dusa' and BG83 avocado genotypes throughout experiments 2 and 3 , respectively. In each experiment, data from the two trials were joined in the same plot. Asterisks indicate significant differences between inoculated and control treatments $(P<0.05)$. Each data point represents the mean $( \pm$ standard error; $n=8$ to 18). 
(TT-16 and TT-21, $P=0.001$; Dusa, $P=0.006$ ), showing maximum levels close to 1 month after inoculation (Figs. 3E and 4E). In TT-31 plants, the physiological parameters displayed tendencies similar to those described for TT-16 and TT-21 genotypes but with a 2-week delay (Fig. 3B, D, and F). In the tolerant genotype BG83, no significant changes in any of the chlorophyll fluorescence parameters were observed during disease progression (Fig. 4B, D, and F). No symptoms of leaf chlorosis were observed in any of the genotypes studied under either treatment, made evident as a lack of significant differences in SPAD index between treatments and among genotypes (Figs. 3G and $\mathrm{H}$ and $4 \mathrm{G}$ and $\mathrm{H}$ ) throughout the experimental courses.

Imaging GFP-tagged $R$. necatrix strain to visualize aboveground symptoms. Roots of infected plants at stage 1 showing a reduction of $F_{\mathrm{v}}^{\prime} / F_{\mathrm{m}}{ }^{\prime}$ (i.e., 10 to 15 days postinoculation) exhibited external colonization of $R$. necatrix mycelium but the degree of root colonization varied among individuals. $R$. necatrix was observed either colonizing most of the root surface as a diffuse mycelium (Fig. 5A) or restricted to several points along the root system (Fig. 5B). The CLSM visualization of transverse sections of young root samples possessing primary growth and covered with mycelia revealed the presence of mycelia aggregates penetrating into root tissues followed by hyphae proliferation (Fig. 6A), resulting in the invasion of both epidermal and cortical cells (data not shown). Mycelia were also observed invading roots displaying secondary growth. In these cases, individual hyphae were visualized growing through secondary xylem vessels (Fig. 6B). At this stage, no mycelia were observed in the transverse sections of roots located in the proximity of the collar (Fig. 6C).
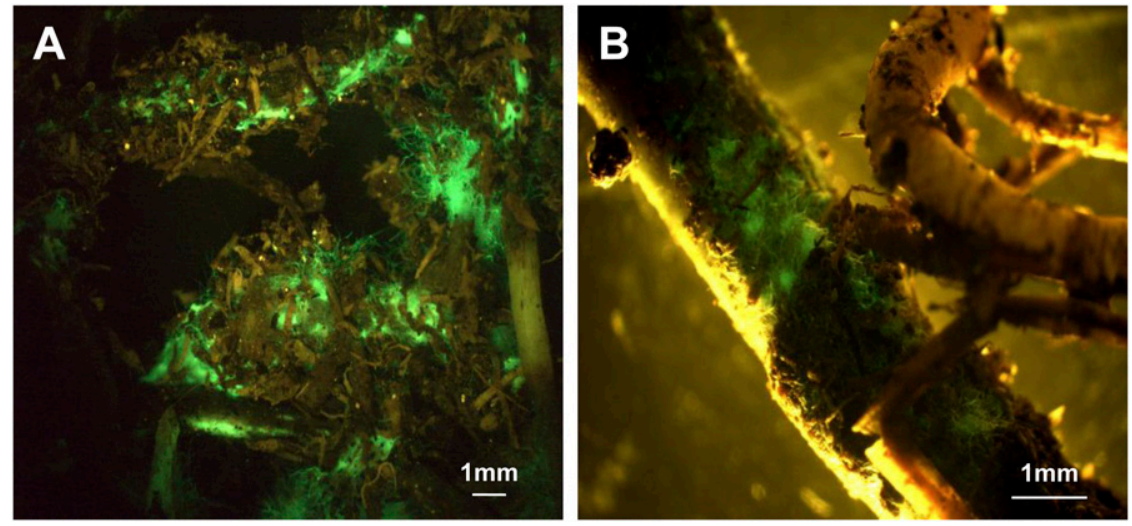

Fig. 5. In situ visualization of Rosellinia necatrix CH53-gfp mycelia colonizing the root surface of avocado plantlets without aerial symptoms. The green fluorescence emitted from $R$. necatrix CH53-gfp was visualized using a fluorescence microscope equipped with filter blocks, with spectral properties matching those of enhanced green fluorescent protein (GFP $488 \mathrm{~nm}$ excitation and emission from 500 to $560 \mathrm{~nm}$ ). A, External proliferation of the hyphae as a diffuse mycelium along the root system. B, External colonization of the hyphae as mycelial aggregates restricted to several points along the root system.
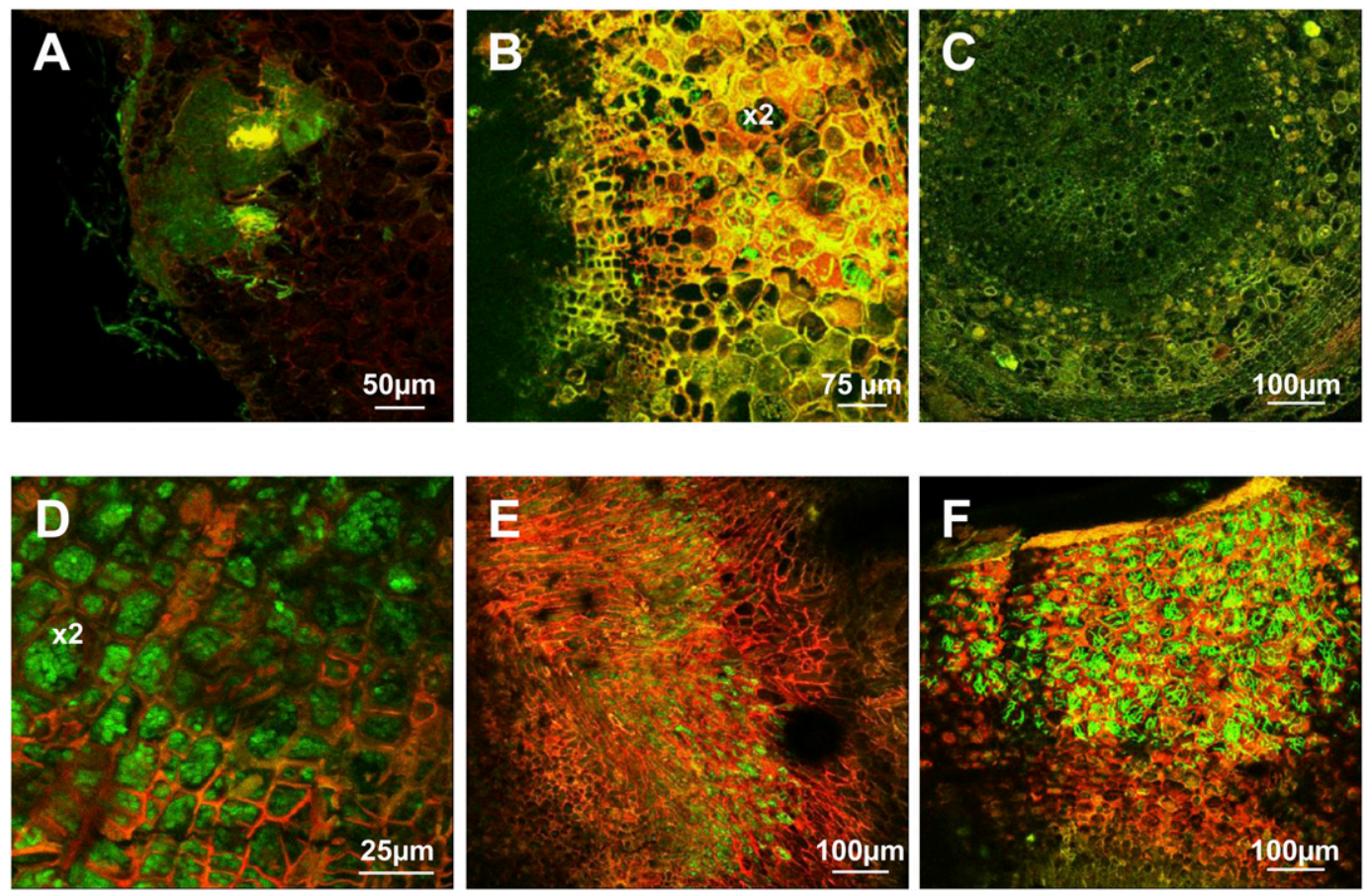

Fig. 6. Confocal laser-scanning microscopy (CLSM) images of the radial sections of avocado roots infected with Rosellinia necatrix CH53-gfp from plants at A, B, and C, stage 1 and D, stage 2. E and F, CLSM images of the radial section of collar and stem from plants at stage 2. A, Penetration and proliferation of $R$. necatrix mycelia throughout the root cortex. B, R. necatrix hyphae growing throughout the secondary xylem $(\times 2)$ vessels of avocado roots with secondary growth. C, Radial sections of avocado roots located in the proximity of the collar from 6-month-old avocado plantlets infected with $R$. necatrix; roots with secondary growth, in which the presence of the fungus could not be detected. D, Detailed image of secondary xylem vessels collapsed from the fungal hyphae. $R$. necatrix mycelia invading undifferentiated cells conforming $\mathrm{E}$, the collar and $\mathrm{F}$, the periderm of the stem base located at $0.5 \mathrm{~cm}$ above the collar. 
To compare $R$. necatrix root invasion at stage 1 (Fig. 1) with advanced stages at which aerial symptoms were observed (stage 2 and 3; Fig. 1), the roots of plants at stages 2 and 3 (i.e., 22 and 27 days postinoculation, respectively) were collected and the areas at the proximity of the root collar were examined. Microscopic visualization of transverse sections through CLSM revealed green fluorescent mycelial aggregations invading most of the cork tissue and the phelloderm, reaching the pith. Hyphal strands were also observed collapsing xylem vessels (Fig. 6E). The visualization of transverse collar sections and stem sections at $0.5 \mathrm{~cm}$ above the collar revealed the invasion of undifferentiated cells on the collar (Fig. 6D) and the profuse invasion of the R. necatrix hyphal network into the periderm of the stem (Fig. 6F). However, in this case, the fungus was not observed colonizing areas bellow the cambium, such as xylem vessels and the pith. No penetration structures, such as mycelial aggregates, were observed on the stem surface.

\section{Discussion}

Herein, we provide the first analysis combining nondestructive chlorophyll fluorescence measurements and CLSM techniques to describe the relationship between the physiological changes in avocado leaves during the first stages of infection and the degree of $R$. necatrix root invasion.

One of the earliest plant responses following pathogen attack is the production of reactive oxygen species in the chloroplast (Berger et al. 2007b; Bolwell et al. 2002; Torres et al. 2006) and the uncoupling or inhibition of the photosystem machinery (Berger et al. 2007b). In the present study, we showed that leaf photochemistry was affected early after root infection with $R$. necatrix, as reflected by the decrease in the trapping efficiency of PSII $\left(F_{\mathrm{v}}{ }^{\prime} / F_{\mathrm{m}}{ }^{\prime}\right)$ before any visible symptoms were observed. This parameter reflects the changes in nonphotochemical processes affecting maximum PSII efficiency (Baker et al. 2007), which likely involves some degree of photo-inactivation of the PSII reaction centers (Havaux et al. 1991; Martínez-Ferri et al. 2000).

These changes could be associated with the localized effects of fungal phytotoxic metabolites on the roots or phytotoxic effects on the leaves induced through mobile signals, as reported for other woody plants (Fleischmann et al. 2005; Grimmer et al. 2012). $R$. necatrix strains produce phytotoxic metabolites such as cytochalasin E (Chen 1964; Cole and Cox 1981; Kimura et al. 1989; Kshirsagar et al. 2001), rosellichalasin (Kimura et al. 1989), rosellinic acid, rosnecatrone, and diketopiperazines (Edwards et al. 2001), although roles for these compounds in pathogenicity remain unclear. Disease progression was not affected in plants inoculated with $R$. necatrix mutants not producing cytochalasin E, suggesting that this toxic compound is not a major $R$. necatrix pathogenicity factor (Kanematsu et al. 1997).

Previous studies have reported that the primary mechanisms of woody plants in response to root pathogen infections are associated with the impairment of water relations and the limitation of photosynthesis (Clemenz et al. 2008; Fleischmann et al. 2005). In the present study, we observed an early and significant decrease in $F_{\mathrm{s}} / F_{0}$ ratio before any aerial symptoms were observed. Studies have previously shown that $F_{\mathrm{S}} / F_{0}$ is an excellent indicator of declining stomatal conductance, $\mathrm{CO}_{2}$ assimilation, and NPQ generation during mild water stress, thereby providing an adequate method for the early detection of water stress (Flexas et al. 2002). The $F_{\mathrm{s}} / F_{0}$ parameter consolidates the cascade of consequences derived from stomatal limitations of photosynthesis. Thus, the sustained decrease in $F_{\mathrm{S}} / F_{0}$ associated with the increase in NPQ under nonlimiting water availability suggests that $R$. necatrix infection could be also associated with stomatal limitations of photosynthesis in avocado plantlets before aerial symptoms are evident (Fig. 1, stage 1). CLSM images revealed that these changes were concomitant with the initial invasion of the root vascular system, which might affect water flow toward the aerial ground parts of the plant. These results are consistent with previous studies on avocado plants infected with the root pathogen Phytophthora cinnamomi Rands, in which root infection impaired water transport between roots and leaves (Sterne et al. 1978). However, further studies, including the measurement of water relations (i.e., plant water status, gas exchange, and stomatal conductance), are necessary to clarify the relative contribution of stomatal limitations of photosynthesis in WRR disease development.

Notably, during the early stages of infection, different degrees of root colonization were observed but no mycelia were detected at the root collar or at the roots located in the proximity of the collar. These differences in the degree of mycelial colonization along the root did not translate into proportional differences in chlorophyll fluorescence parameters, suggesting that photosynthetic impairment is not dependent on the degree of mycelial invasion of the lateral root system. This result also demonstrates the reduced contribution of infected lateral roots to the transpirational stream compared with the roots close to the root collar and suggests a counteracting effect of noninvaded roots.

Despite the presence of the fungus along the root system, the decrease in chlorophyll fluorescence parameters observed in TT-31 was delayed 2 weeks compared with TT-16 and TT-21. These results indicate that TT-31 might have a lower susceptibility to $R$. necatrix, which would not be associated with constitutive differences in the growth or biomass partitioning among genotypes. This response seems to be exclusive to susceptible genotypes because no variation in the photosynthetic parameters was observed in the tolerant genotype (BG83). Thus, the results of the present study show that chlorophyll fluorescence may potentially represent a useful, nondestructive technique for disease diagnosis which could support the discarding of highly susceptible genotypes in breeding programs in a short period of time. With this method, we would discard TT-16 and TT-21 as possible candidates for the selection program at 10 to 15 days postinoculation.

The absence of changes at the photosynthetic level in the tolerant genotype (BG83) despite of the presence of aerial symptoms suggests that avocado tolerance to $R$. necatrix could be associated with mechanisms conferring a higher robustness to the photosynthetic machinery. Moreover, the comparatively higher root investment observed in BG83 could indicate a fast root growth rate or root turnover, which can counteract the necrotic effects of $R$. necatrix in the root system, as has been reported in apple genotypes tolerant to replant disease (Atucha et al. 2014). To explore these issues in more depth, further studies with tolerant genotypes are necessary.

Once the aerial symptoms became obvious (Fig. 1, stage 2), the observed wilting suggested the appearance of water stress, which was concomitant with pronounced physiological differences in susceptible genotypes. These changes were associated with the profuse invasion of the root vascular system and the fungal colonization of the root collar, obstructing water flow to the aerial parts of the plant. Taken together, these results suggest that visible symptoms of WRR are associated with a mechanical effect on the main vascular system.

Plant desiccation and death occurred within a few days after the appearance of wilting symptoms in all genotypes. The lack of chlorosis, as shown by the SPAD index, might indicate that the potential effects of toxins during disease progression did not result in losses of leaf integrity and chlorophyll photo-oxidation (Bolwell et al. 2002) in contrast to the effects reported for other fungal toxins (Kim et al. 2010).

In conclusion, this study is the first to provide information on the physiological changes that occur during the initial stages of $R$. necatrix infection on avocado roots prior to the appearance of aboveground visible symptoms in susceptible and tolerant genotypes. This knowledge is essential for further studies of the avocado- $R$. necatrix interaction system. Thus, studies examining avocado gene expression profiles in contrasting disease reaction genotypes would be addressed to obtain a better understanding of the molecular interaction between the host and pathogen, which is crucial for the development of new tools to control disease. Moreover, the detection of $R$. necatrix infection on roots before aerial symptoms are observed would facilitate the application of control strategies during the early stages of the infection, avoiding tree death and further disease spread, which are major problems to overcome when managing WRR disease. However, the use of 
chlorophyll fluorescence as a diagnosis tool in the field needs further investigation to test its validity. Under field conditions, leaf photochemistry can be affected by several environmental factors (i.e., water, $\mathrm{T}$, and nutrients) and it would be necessary to assure that any other type of stress is not operating in plants under evaluation.

\section{Acknowledgments}

This research was supported through funding from the AVA201301.13 project co-financed through the Junta de Andalucía and European Union (FEDER). M. C. Pliego and M. T. Ariza are currently supported by IFAPA Junta de Andalucía (20\%) and by the Programa Operativo Fondo Social Europeo (FSE) de Andalucía 2007-2013 (80\%) under the topic "Andalucía se mueve con Europa". We thank D. Navas for his technical support in CLSM studies, M. Carrera for her technical assistance, and three anonymous referees for their critical comments on a previous draft of this manuscript.

\section{Literature Cited}

Alvarez, J. P., Pekker, I., Goldshmidt, A., Blum, E., Amsellem, Z., and Eshed, Y. 2006. Endogenous and synthetic microRNAs stimulate simultaneous, efficient, and localized regulation of multiple targets in diverse species. Plant Cell 18: 1134-1151.

Atucha, A., Emmett, B., and Bauerle, T. L. 2014. Growth rate of fine root systems influences rootstock tolerance to replant disease. Plant Soil 376:337-346.

Baker, N. R., Harbinson, J., and Kramer, D. M. 2007. Determining the limitations and regulation of photosynthetic energy transduction in leaves. Plant Cell Environ. 30:1107-1125.

Barceló-Muñoz, A., Zea-Bonilla, T., Jurado-Valle, I., Imbroda-Solano, I., Vidoy-Mercado, I., Pliego-Alfaro, F., and López-Herrera, C. J. 2007. Programa de selección de portainjertos de aguacate tolerantes a la podredumbre blanca causada por Rosellinia necatrix en el Sur de España (1999-2007). Pages 537-541 in: Proc. VI World Avocado Congress, Viña del Mar, CL.

Bauriegel, E., and Herppich, W. B. 2014. Hyperspectral and chlorophyll fluorescence imaging for early detection of plant diseases, with special reference to Fusarium spp. infections on wheat. Agriculture 4:32-57.

Berger, S., Benediktyova, Z., Matous, K., Bonfig, K. B., Mueller, M. J., Nedbal, L., and Roitsch, T. 2007a. Visualization of dynamics of plant-pathogen interaction by novel combination of chlorophyll fluorescence imaging and statistical analysis: Differential effects of virulent and avirulent strains of $P$. syringae and of oxylipins on A. thaliana. J. Exp. Bot. 58:797-806.

Berger, S., Sinha, A., Thomas, K., and Roitsch, T. 2007b. Plant physiology meets phytopathology: Plant primary metabolism and plant-pathogen interactions. J. Exp. Bot. 58:4019-4026.

Bilger, W., and Björkman, O. 1990. Role of the xanthophyll cycle in photoprotection elucidated by measurements of light-induced absorbance changes, fluorescence and photosynthesis in leaves of Hedera canariensis. Photosynth. Res. 25:173-185.

Bolwell, G. P., Bindschedler, L. V., Blee, K. A., Butt, V. S., Davies, D. R., Gardner, S. L., Gerrish, C., and Minibayeva, F. 2002. The apoplastic oxidative burst in response to biotic stress in plants: A three-component system. J. Exp. Bot. 53:1367-1376.

Broadbent, P., and Gollnow, B. I. 1993. Selecting disease-resistant citrus rootstocks. Aust. J. Exp. Agric. 33:775-780.

Brummer, M., Arend, M., Fromm, J., Schlenzig, A., and Oßwald, W. F. 2002. Ultrastructural changes and immunocytochemical localization of the elicitin quercinin in Quercus robur L. roots infected with Phytophthora quercina. Physiol. Mol. Plant Pathol. 61:109-120.

Calderón, R., Lucena, C., Trapero-Casas, J. L., Zarco-Tejada, P. J., and NavasCortés, J. A. 2014. Soil temperature determines the reaction of olive cultivars to Verticillium dahliae pathotypes. PLoS One 9(10):e110664, doi: 10.1371/journal.pone.0110664.

Chen, Y. 1964. Studies on the metabolic products of Rosellinia necatrix Berlesse. Part II. The structure of rosellinic acid. Agric. Biol. Chem. 28:431-435.

Clemenz, C., Fleischmann, F., Haberle, K. H., Matyssek, R., and Oßwald, W. 2008. Photosynthetic and leaf water potential responses of Alnus glutinosa saplings to stem-base inoculation with Phytophthora alni subsp. alni. Tree Physiol. 28:1703-1711

Cole, R. J., and Cox, R. H. 1981. The cytochalasins. Pages 264-343 in: Handbook of Toxic Fungal Metabolites. R. J. Cole, ed. Academic Press, London.

Dadmal, S. M., Pawar, N. P., Kale, K. B., and Shivankar, S. K. 2002. Screening of citrus rootstock against leaf miner Phyllocnistis citrella Stainton. Insect Environ. 8:143-144.

Edwards, R. L., Maitland, D. J., Scowen, I. J., Teixeira de Sousa, A. J., and Whalley, A. J. S. 2001. Metabolites of the higher fungi. Part 32. Rosnecatrone, a phytotoxic bicyclo[4.1.0]hept-3-en-2-one from the fungus Rosellinia necatrix Prill. J. Chem. Soc. Perk. T. 1:537-542.

Fleischmann, F., Göttlein, A., Rodenkirchen, H., Lütz, C., and Oßwald, W. F. 2004. Biomass, nutrient and pigment content of beech (Fagus sylvatica) saplings infected with Phytophthora citricola, P. cambivora, P. pseudosyringae and P. undulata. For. Pathol. 34:79-92.

Fleischmann, F., Koehl, J., Portz, R., Beltrame, A. B., and Oßwald, W. 2005. Physiological changes of Fagus sylvatica seedlings infected with Phytophthora citricola and the contribution of its elicitin 'citricolin' to pathogenesis. Plant Biol. 7:650-658.

Fleischmann, F., Schneider, D., Matyssek, R., and Oßwald, W. F. 2002 Investigations on net $\mathrm{CO} 2$ assimilation, transpiration and root growth of Fagus sylvatica infested with four different Phytophthora species. Plant Biol. 4:144-152.

Flexas, J., Escalona, J. M., Evain, S., Gulías, J., Moya, I., Osmond, C. B., and Medrano, H. 2002. Steady-state chlorophyll fluorescence (Fs) measurements as a tool to follow variations of net $\mathrm{CO}_{2}$ assimilation and stomatal conductance during water-stress in $\mathrm{C}_{3}$ plants. Physiol. Plant. 114:231-240.

Freeman, S., and Sztejnberg, A. 1992. Rosellinia. Pages 71-73 in: Methods for Research on Soilborne Phytopathogenic Fungi. L. L. Singleton, J. D. Mihail, and C. M. Rush, eds. American Phytopathological Society Press, St. Paul, MN.

Genty, B., Briantais, J., and Baker, N. 1989. The relationship between the quantum yield of photosynthetic electron transport and photochemical quenching of chlorophyll fluorescence. Biochim. Biophys. Acta 990:87-92.

Grimmer, M. K., Foulkes, M. J., and Paveley, N. D. 2012. Foliar pathogenesis and plant water relations; a review. J. Exp. Bot. 63:4321-4331

Havaux, M., Strasser, R. J., and Greppin, H. 1991. A theoretical and experimental analysis of the $\mathrm{qP}$ and $\mathrm{qN}$ coefficients of chlorophyll fluorescence quenching and their relation to photochemical and nonphotochemical events. Photosynth. Res. 27:41-55.

Jifon, J. L., Syvertsen, J. P., and Whaley, E. 2005. Growth environment and leaf anatomy affect nondestructive estimates of chlorophyll and nitrogen in Citrus spp. leaves. J. Am. Soc. Hortic. Sci. 130:152-158.

Kanematsu, S., Hayashi, T., and Kudo, A. 1997. Isolation of Rosellinia necatrix mutants with impaired cytochalasin E production and its pathogenicity. Jpn. J. Phytopathol. 63:425-431.

Kim, Y. M., Bouras, N., Kav, N. N. V., and Strelkov, S. E. 2010. Inhibition of photosynthesis and modification of the wheat leaf proteome by Ptr ToxB: A host-specific toxin from the fungal pathogen Pyrenophora tritici-repentis. Proteomics 10:2911-2926.

Kimura, Y., Nakajima, H., and Hamasaki, T. 1989. Structure of Rosellichalasin, a new metabolite produced by Rosellinia necatrix. Agric. Biol. Chem. 53: 1699-1701

Kshirsagar, A., Reid, A. J., McColl, S. M., Saunders, V. A., Whalley, A. J. S., and Evans, E. H. 2001. The effect of fungal metabolites on leaves as detected by chlorophyll fluorescence. New Phytol. 151:451-457.

López-Herrera, C. J., Pérez-Jiménez, R. M., Basallote-Ureba, M. J., Zea-Bonilla, T., and Melero-Vara, J. M. 1999. Loss of viability of Dematophora necatrix in solarized soils. Eur. J. Plant Pathol. 105:571-576.

López-Herrera, C. J., and Zea-Bonilla, T. 2007. Effects of benomil, carbendazim, fluazinan and thiophanate methyl on white root rot of avocado. Crop Prot. 26: 1186-1192.

Markwell, J., Ostermann, J. C., and Mitchell, J. L. 1995. Calibration of the Minolta SPAD-502 leaf chlorophyll meter. Photosynth. Res. 46:467-472.

Martínez-Ferri, E., Balaguer, L., Valladares, F., Chico, J. M., and Manrique, E. 2000. Energy dissipation in drought-avoiding and drought-tolerant tree species at midday during the Mediterranean summer. Tree Physiol. 20:131-138.

Pliego, C., Kanematsu, S., Ruano-Rosa, D., De Vicente, A., López-Herrera, C. Cazorla, F. M., and Ramos, C. 2009. GFP sheds light on the infection process of avocado roots by Rosellinia necatrix. Fungal Genet. Biol. 46:137-145.

Pliego, C., López-Herrera, C., Ramos, C., and Cazorla, F. M. 2012. Developing tools to unravel the biological secrets of Rosellinia necatrix, an emergen threat to woody crops. Mol. Plant Pathol. 13:226-239.

Pliego-Alfaro, F., Barceló-Muñoz, A., López-Gómez, R., Ibarra-Laclette, L., Herrera-Estrella, L., Palomo-Ríos, E., Mercado, J. A., and Litz, R. E. 2013. Biotechnology. Pages 268-300 in: The Avocado: Botany, Production and Uses, 2nd ed. A. W. Whiley, B. Schaffer, and B. N. Wolstenholme. eds. CABI Publishing, Oxon, UK.

Raj, H., and Sharma, S. D. 2009. Integration of soil solarization and chemical sterilization with beneficial microorganisms for control of white root rot and growth of nursery apple. Sci. Hortic. (Amsterdam) 119:126-131.

Ruano-Rosa, D., Schena, L., Ippolito, A., and López-Herrera, C. J. 2007. Comparison of conventional and molecular methods for the detection of Rosellinia necatrix in avocado orchards in southern Spain. Plant Pathol. 56:251-256.

Schena, L., and Ippolito, A. 2003. Rapid and sensitive detection of Rosellinia necatrix in roots and soils by real time Scorpion-PCR. J. Plant Pathol. 85:15-25.

Schena, L., Nigro, F., and Ippolito, A. 2002. Identification and detection of Rosellinia necatrix by conventional and real-time Scorpion-PCR. Eur. J. Plant Pathol. 108:355-366.

Schreiber, U., Bilger, W., Hormann, H., and Neubauer, C. 1998. Chlorophyll fluorescence as a diagnostic tool: The basics and some aspects of practical relevance. Pages 320-326 in: Photosynthesis: A Comprehensive Treatise. A. S. Raghavendra, ed. Cambridge University Press, Cambridge, UK.

Schreiber, U., Bilger, W., and Neubauer, C. 1994. Chlorophyll fluorescence as a non-intrusive indicator for rapid assessment of in vivo photosynthesis. Pages 49-70 in: Ecophysiology of Photosynthesis. E. D. Schulze and M. M Caldwell, eds. Springer-Verlag, Berlin.

Sterne, R. E., Kaufmann, M. R., and Zentmyer, G. A. 1978. Effect of Phytophthora root rot on water relations of avocado: Interpretation with a water transport model. Phytopathology 68:595-602. 
Sugimoto, K. 2002. Fluazinam (Frowncide ${ }^{\circledR}$ ) a novel and effective method of application against white and violet root rot. Agrochem. Jpn. 80:14-16.

Sztejnberg, A., and Madar, Z. 1980. Host range of Dematophora necatrix, the cause of white root rot disease in fruit trees. Plant Dis. 64:662-664.

Tavakkoli, E., Rengasamy, P., and McDonald, G. K. 2010. High concentrations of $\mathrm{Na}^{+}$and $\mathrm{Cl}^{-}$ions in soil solution have simultaneous detrimental effects on growth of faba bean under salinity stress. J. Exp. Bot. 61:4449-4459.

ten Hoopen, G. M., and Krauss, U. 2006. Biology and control of Rosellinia bunodes, Rosellinia necatrix and Rosellinia pepo: A review. Crop Prot. 25:89-107.

Torres, M. A., Jones, J. D. G., and Dangl, J. L. 2006. Reactive oxygen species signaling in response to pathogens. Plant Physiol. 141:373-378.
Trapero Muñoz, S., Hervalejo García, A., Jiménez Pérez, M., Boyero, J. R., Vela, J. M., and Martínez-Ferri, E. 2008. Effects of rootstock and flushing on the incidence of three insects on 'Clementine de Nules' citrus trees. Environ. Entomol. 37:1531-1537.

Uddling, J., Gelang-Alfredsson, J., Piikki, K., and Pleijel, H. 2007. Evaluating the relationship between leaf chlorophyll concentration and SPAD-502 chlorophyll meter readings. Photosynth. Res. 91:37-46.

Verdejo-Lucas, S., Galeano, M., Sorribas, F. J., Forner, J. B., and Alcaide, A. 2003. Effect on resistance to Tylenchulus semipenetrans of hybrid citrus rootstocks subjected to continuous exposure to high population densities of the nematode. Eur. J. Plant Pathol. 109:427-433. 